\title{
Incomplete Treatment of Neonatal Pneumonia Causing Giant Pneumatocele in a 45-Day Infant
}

\author{
Elie Choueiry ${ }^{\mathrm{a}, \mathrm{b}}$, Sandy Diab ${ }^{\mathrm{b}}$, Carla El Haber ${ }^{\mathrm{b}, ~ e}$, Paul Henry Torbey ${ }^{\mathrm{b}, \mathrm{c}}$, \\ Zaki Ghorayeb $^{\mathrm{d}}$, Bernard Gerbaka ${ }^{\mathrm{a}, \mathrm{b}}$
}

\begin{abstract}
We report the case of giant pneumatocele due to an incomplete treatment of neonatal pneumonia in a 45-day infant. Two pneumatoceles were formed in the seventh day of pneumonia previously treated for 2 days, and none of them was under tension; however, the size gradually increased. The patient remained asymptomatic. All cultures taken failed to isolate the causing bacteria. Intravenous antibiotics resulted in a successful treatment. The purpose of this case is to highlight the importance of adequate treatment when neonatal pneumonia is highly suspected with normal radiological findings, to search for pneumatoceles as a complication of inadequately treated pneumonia and to rule out congenital malformations in case of pulmonary cyst.
\end{abstract}

Keywords: Pneumatocele; Pneumonia; Infant

\section{Introduction}

Pulmonary pneumatoceles are thin-walled, air-filled cysts that develop within the lung parenchyma as a complication of pneumonia. They can also result from non-infectious etiologies such as trauma and positive pressure ventilation [1]. There are limited data about post-infectious pulmonary cysts in newborns. They can be single emphysematous lesion, but are more often multiple. Most often, pneumatocele is commonly seen in infants and young children as a complication of Staphylococcus aureus pneumonia [2,3] but can result

Manuscript submitted December 16, 2016, accepted February 15, 2017

aPediatric Intensive Care Unit, Department of Pediatrics, Hotel Dieu De France Hospital, Beirut, Lebanon

${ }^{b}$ Department of Pediatrics, Hotel Dieu De France Hospital, Beirut, Lebanon

cPediatric Pulmonary Unit, Department of Pediatrics, Hotel Dieu De France Hospital, Beirut, Lebanon

${ }^{\mathrm{d} D e p a r t m e n t}$ of Pediatric Surgery, Hotel Dieu De France Hospital, Beirut, Lebanon

${ }^{e}$ Corresponding Author: Carla El Haber, Department of Pediatrics, Hotel Dieu De France Hospital, Beirut, Lebanon. Email: Carla_haber@hotmail.com

doi: https://doi.org/10.14740/ijcp265w from a variety of bacteriologic infections such as Escherichia coli pneumonia [4]. Congenital and acquired conditions (i.e. pulmonary sequestration, bronchogenic cyst, and congenital lobar emphysema) should be considered in the differential diagnosis of cystic pulmonary lesions in newborns [5]. We report a 45-day-old Lebanese male infant, who presented a pneumatocele resulting from an incompletely treated bacterial pneumonia. This case highlights the importance of maintaining a high index of suspicion of pneumonia in neonates even with normal radiological findings and searching for pneumatoceles and other complications when the pneumonia is inadequately treated.

\section{Case Report}

We report the case of a 45-day-old male infant who presented to our emergency department in October 2015 with irritability, hypotonia and high grade fever $\left(>39^{\circ} \mathrm{C}\right)$ starting $24 \mathrm{~h}$ prior to admission. He is one of two non-identical term twins born by in vitro fertilization at 37 weeks by C-section. Apgar score was 9 at $1 \mathrm{~min}$ of life and 10 at 5 and $10 \mathrm{~min}$ of life. Amniotic liquid was clear, immediate cry and no infectious risk at birth. Birth weight was $2,450 \mathrm{~g}$. He had no relevant past medical history. He was exclusively breastfed and had received his first dose of hepatitis B at birth and his second dose at 1 month. He had one sister of 4 years old in good health, and none of his parents was sick at home.

On admission, vital signs revealed a heart rate of $155 / \mathrm{min}$, respiratory rate of 38 breaths/min, CRT $<3 \mathrm{~s}$, an axillary temperature of $39^{\circ} \mathrm{C}$ and weight of $4,540 \mathrm{~g}$. He was hypotonic and pale, archaic reflexes were present and normal, and anterior fontanel was normotensive. There were no signs of dyspnea, respiratory distress, cyanosis and dehydration. Chest auscultation was symmetric, with no heart murmur. Abdomen was soft, non-distended, no hepatosplenomegaly and no mass palpated were shown. Genital organs were normal.

Full infectious investigations with lumbar puncture were done, except for a sputum culture. Initial full blood count revealed packed cell volume of $28 \%$, white cell count of 25,200 / $\mathrm{mm}^{3}$ with $53 \%$ neutrophils, $33 \%$ lymphocytes, $12 \%$ monocytes, absolute platelet count of $626,000 / \mathrm{mm}^{3}$, and a C-reactive protein of $33.3 \mathrm{mg} / \mathrm{dL}$. Urine analysis showed $2 \mathrm{WBC}, 1$ $\mathrm{RBC}$, sterile. Cerebrospinal fluid analysis revealed $1 \mathrm{WBC}, 15$ 


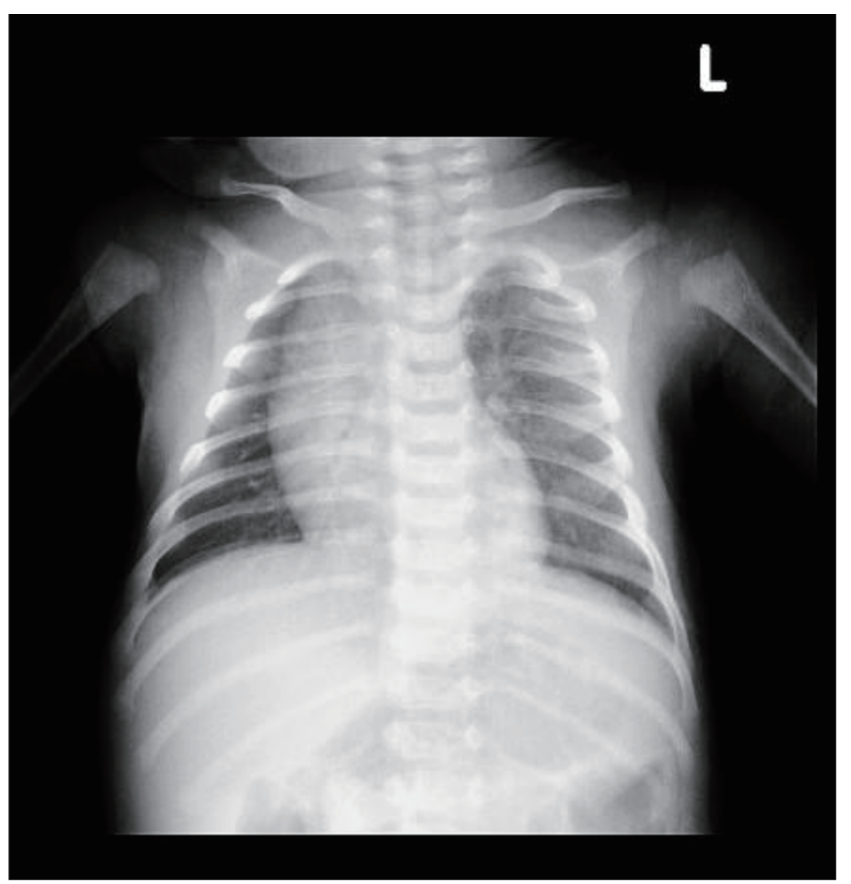

Figure 1. Initial chest $\mathrm{X}$-ray with normal findings.
RBC, $0.85 \mathrm{~g} / \mathrm{L}$ proteins, and $4 \mathrm{mmol} / \mathrm{L}$ glucose. The chest $\mathrm{X}-$ ray was normal not revealing any alveolar condensation (Fig. 1). The patient was placed on intravenous antibiotics for an infectious risk (ampicillin $200 \mathrm{mg} / \mathrm{kg}$ /day q8h IV and cefotaxime $200 \mathrm{mg} / \mathrm{kg} /$ day q8h IV).

Antibiotics were stopped 2 days after admission and the patient was discharged, although still febrile $\left(38.2^{\circ} \mathrm{C}\right)$, for a presumed viral infection since none of the cultures (blood, urine and cerebrospinal fluid cultures) showed bacteria.

A week later, he went back to the clinic for follow-up because of a persistent low grade fever associated to irritability, lethargy and vomiting. He was transferred to the emergency department and admitted to the hospital. Vital signs revealed a heart rate of $160 / \mathrm{min}$, respiratory rate of 43 breaths $/ \mathrm{min}$ and an axillary temperature of $38.1^{\circ} \mathrm{C}$. Weight for age and other anthropometric parameters were normal for age but the weight upon consultation was 4,195 $\mathrm{g}$ (he had lost $350 \mathrm{~g}$ in 1 week). Examining the infant upon his presentation to the hospital, he did not look ill, despite being mildly pale. Neurological exam was normal, and there were no signs of dyspnea, respiratory distress, cyanosis or dehydration. Auscultation was asymmetric with decreased murmur on the left side.

Full blood count revealed packed cell volume of $25 \%$, white cell count of $17,700 / \mathrm{mm}^{3}$ with $36 \%$ neutrophils, $46 \%$ lymphocytes, $18 \%$ monocytes, absolute platelet count of

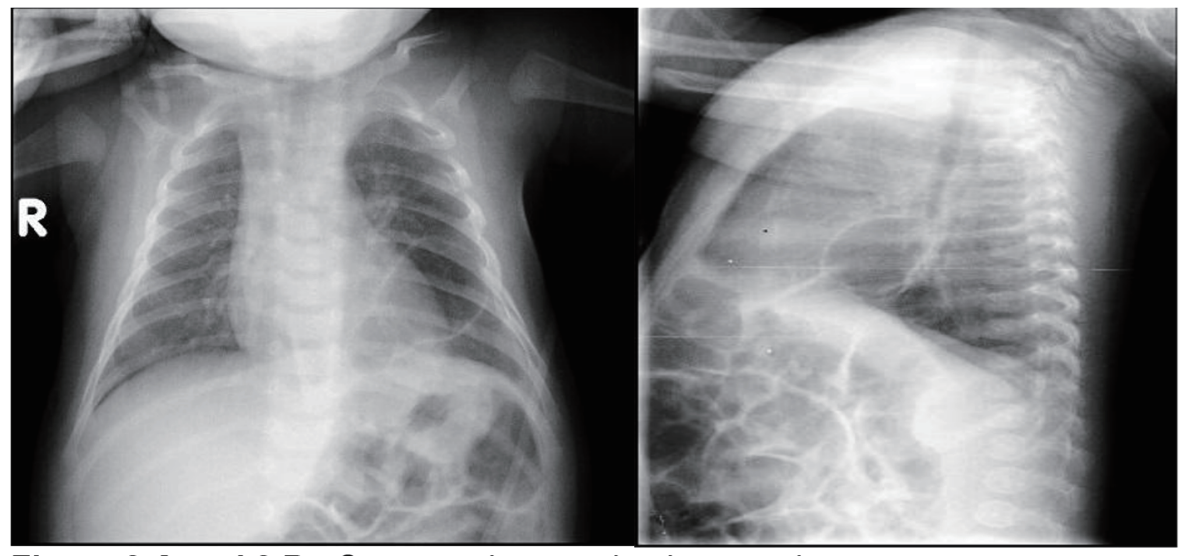

Figure 2. Suspected aerated pulmonary image.

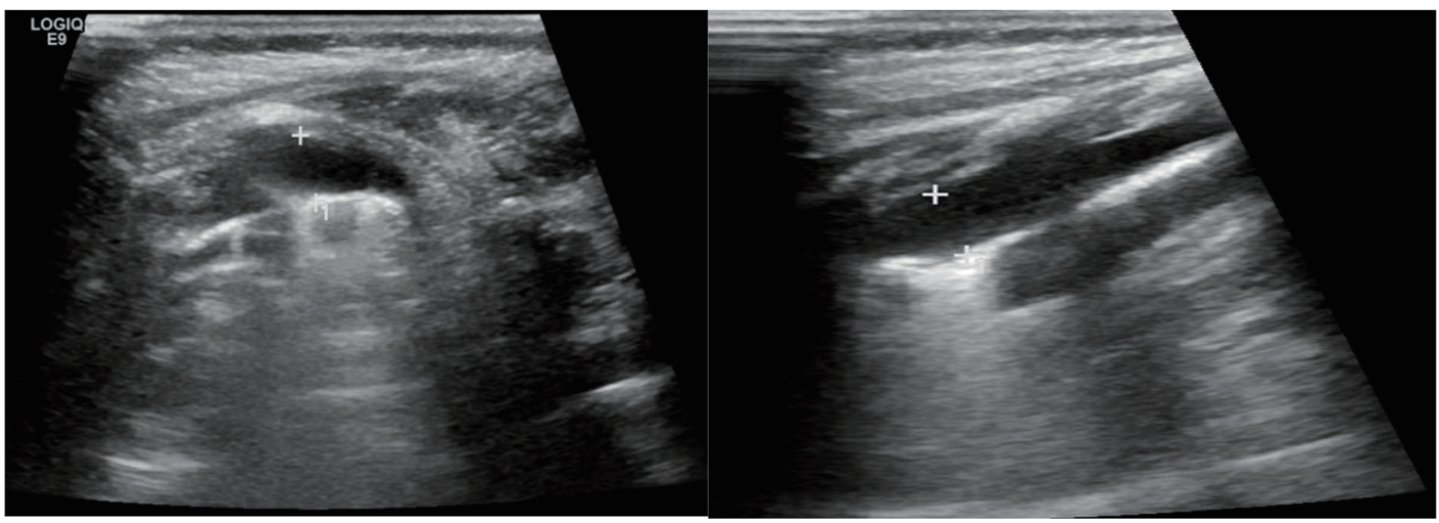

Figure 3. Left posterolateral pleural effusion on abdominal ultrasound. 


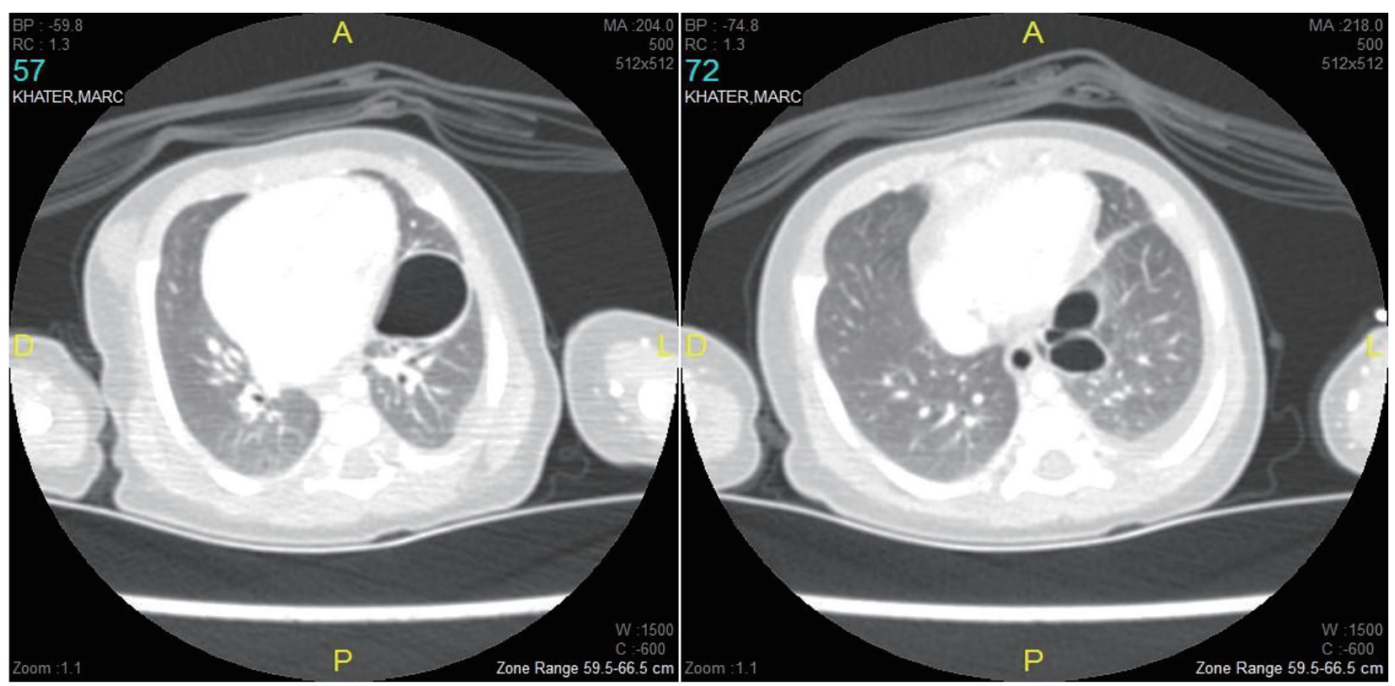

Figure 4. Chest CT scan showing two cystic images in the upper segment of the lower left lobe with a left small pleural effusion.

$856,000 / \mathrm{mm}^{3}$ and a C-reactive protein less than $3.5 \mathrm{mg} / \mathrm{dL}$. A sample for sputum culture taken at the peak of pyrexia showed $>25$ epithelial cells, $<25 \mathrm{WBC}$ and was sterile. Blood culture was also taken at the peak of pyrexia and was sterile. A chest X-ray was taken and revealed a suspected aerated pulmonary image (Fig. 2) and an initial diagnosis of suspected diaphragmatic hernia was established. An abdominal ultrasound showed a left posterolateral pleural effusion with a pulmonary opacification of the intermediate segment of the left inferior lobe (Fig. 3). A chest CT scan revealed two cystic images in the upper segment of the lower left lobe of 28 and $14 \mathrm{~mm}$, respectively, presenting a thick wall associated with a strip of a lingular atelectasis and creeping into the great left scissure, with a left small pleural effusion (Fig. 4). A diagnosis of pneumatocele resulting probably from an untreated bacterial pneumonia was made, although cultures (blood and sputum) did not show the responsible bacteria. The patient was placed

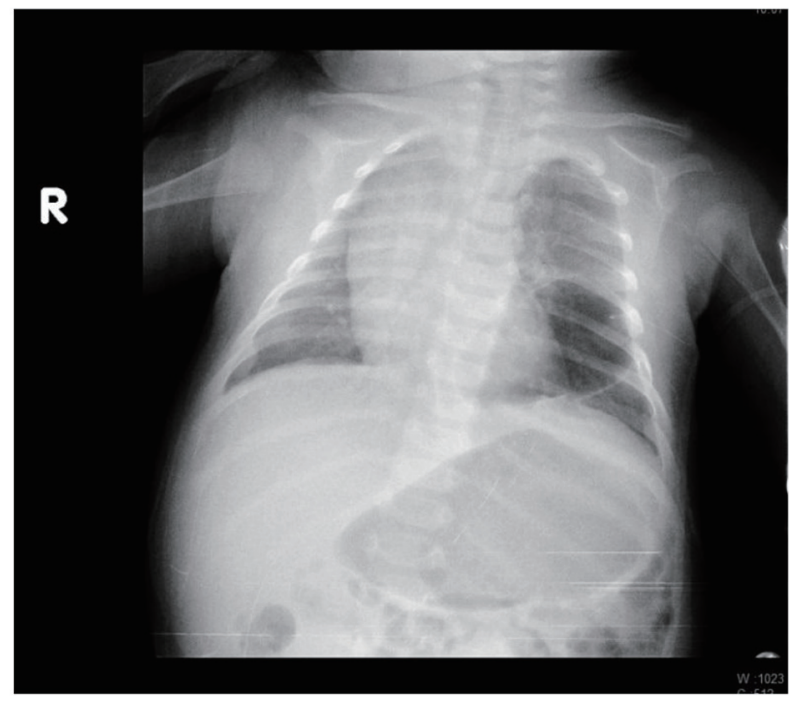

Figure 5. The pneumatocele increased in size. on intravenous antibiotics: teicoplanin $10 \mathrm{mg} / \mathrm{kg} / 12 \mathrm{~h} \mathrm{IV}$ and ceftriaxone $75 \mathrm{mg} / \mathrm{kg} /$ day IV, and intravenous infusion of glucose $5 \%$ and electrolytes.

At day 20 of the pneumonia, the patient became tachypneic with asynchronous thoraco abdominal swing. Chest radiography revealed an enlargement of the pneumatoceles without causing any mediastinal shift (Fig. 5). No hypoxia was detected and there was no need for oxygenation. The patient was thus put under close continuous vital monitoring without any need of admission to the intensive care unit. Two days later, we noted a clinical and radiologic improvement: pneumatoceles began to diminish in size.

He was discharged after 21 days of antibiotic treatment, although he remained afebrile after the first day of the admission.

As a follow-up, subsequent clinical and radiologic evaluation revealed no abnormality with a complete regression and return to normal lungs in 45 days period (Fig. 6).

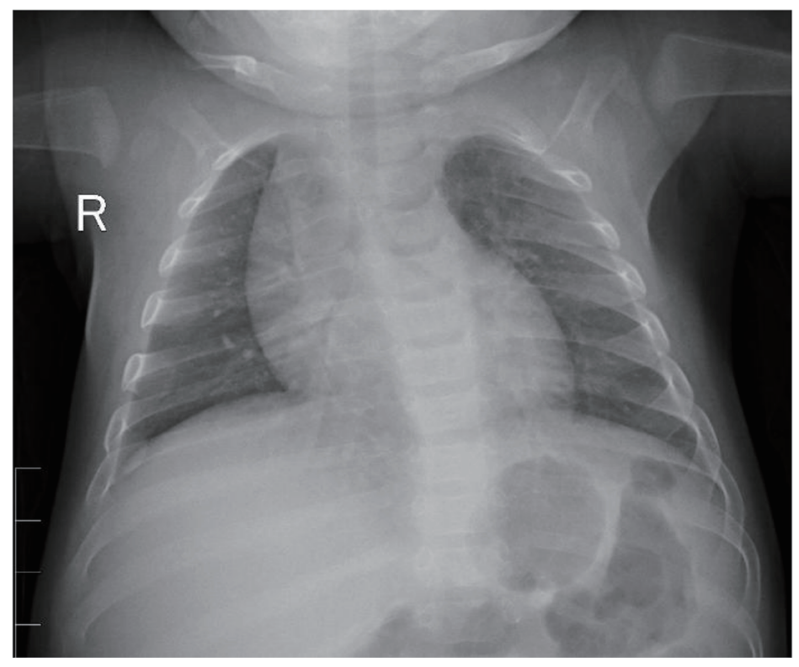

Figure 6. Normal chest X-ray after antibiotic treatment. 


\section{Discussion}

Neonatal pneumonia can be a difficult clinical and radiographic diagnosis: an initial normal chest radiograph in neonates does not eliminate the diagnosis of pneumonia. For that reason all cultures (blood, sputum, urine and CSF in case of fever) should be taken as soon as possible and the treatment initiated along with a continuous clinical evaluation of the infant for further investigations to detect complications such as pneumatoceles (repeat chest X-ray, redo a blood test). Frequently, the child will be covered with antibiotics without positive confirmation of pneumonia. A pulmonary pneumatocele is a frequent radiological finding that can occur during the course of acute pneumonia in children. Pneumatoceles are differentiated from lung abscesses, another radiological finding in the course of pneumonia, due to their tendency to rapidly change in appearance, size and location [2]. The differential diagnosis of a cystic pulmonary lesion includes congenital and acquired conditions such as pulmonary sequestration, bronchogenic cyst, congenital lobar emphysema, congenital cystic adenomatoid malformation, aspiration or bacterial pneumonia with cavitation and Wilson-Mikity syndrome [5]. They are generally observed on the fifth to sixth day of pneumonia, rarely on the initial chest radiograph [6] and they usually subside spontaneously and without sequelae after a few weeks alongside the pneumonic process $[3,6,7]$. Rarely, they may result in complications such as tension, infection, and rupture (pneumothorax) which may be life-threatening. The incidence of the formation of pneumatocele ranges from $2 \%$ to $8 \%$ of all cases of pneumonia in children with incidence up to $85 \%$ in Staphylococcus aureus pneumonias [2,3]. Formation of pneumatocele also occurs with other agents, including Streptococcus pneumoniae, Haemophilus influenzae, Escherichia coli, group A streptococci, Serratia marcescens, Klebsiella pneumoniae, adenovirus, and tuberculosis alongside with Klebsiella pneumonia and Pseudomonas aeruginosa [5, 6]. Two massive tension pneumatoceles were developed while on treatment for post-pneumonic empyema due to Enterobacter gergoviae pneumonia in a 3-month-old Nigerian male [1]. Some cases of post-infectious pneumatocele do not have a definite cause [6]. Non-infectious etiologies of pneumatoceles include the following: trauma, hydrocarbon ingestion, and positive pressure ventilation (especially among premature infants). Postinfectious pneumatoceles probably develop from an initial necrotic parenchymatous lesion, which then becomes cystic due to a check-valve mechanism. The inflammatory exudates within the airways cause bronchiolar obstruction, allowing air to enter in the cystic space but not to leave it with progressive over inflation [5]. In most circumstances, pneumatoceles are asymptomatic. Large pneumatoceles can result in cardiopulmonary insufficiency by causing displacement and compression of the adjacent lung.

Our patient did not have a pulmonary congenital malforma- tion on his first admission and had two pneumatoceles formed on the seventh day of pneumonia, due to an incompletely treated neonatal bacterial pneumonia, both being confined to the left lung; none of them was under tension; however, the size and amount of the air fluid level gradually increased, but our patient had no pneumatocele-related symptoms. The usual medical care of pneumatoceles is the treatment of the underlying pneumonia. This involves administration of broad-spectrum antibiotics directed against the most common causative agent in children, which is $S$. aureus. Surgical approaches are indicated in the case when respiratory function is compromised with tension or infected pneumatoceles and pneumothorax.

In summary, this case highlights the importance of maintaining a high index of suspicion of pneumonia in neonates even with normal radiological findings with a focus on the diagnostic value - and ulterior guided therapeutic approach - of sputum cultures and viral testing to complete the infectious investigation. The initiation of adequate antibiotics is essential to prevent further complications of pneumonia. Clinicians should search for pneumatoceles and other complications when the pneumonia is inadequately treated, and they should always rule out congenital malformations when pulmonary cyst is present on chest X-rays. The wait and see strategy is the cornerstone of the treatment as long as the patient is asymptomatic.

\section{Conflicts of Interest}

The authors have no conflicts of interest to disclose.

\section{References}

1. Kesieme E, Kesieme C, Akpede G, et al. Tension Pneumatocele due to Enterobacter gergoviae Pneumonia: A Case Report. Case Reports in Medicine. 2012;2012.

2. Meyers HI, Jacobson G. Staphylococcal pneumonia in children and adults. Radiology. 1959;72(5):665-671.

3. Ceruti E, Contreras J, Neira M. Staphylococcal pneumonia in childhood. Long-term follow-up including pulmonary function studies. Am J Dis Child. 1971;122(5):386392.

4. Papageorgiou A, Bauer CR, Fletcher BD, Stern L. Klebsiella pneumonia with pneumatocele formation in a newborn infant. Can Med Assoc J. 1973;109(12):1217-1219.

5. Maranella E, Conte E, Di Natale C, Coclite E, Di Fabio S. Disseminated, large-sized neonatal pneumatoceles: the wait-and-see strategy. Pediatr Pulmonol. 2014;49(3):E6971.

6. Amitai I, Mogle P, Godfrey S, Aviad I. Pneumatocele in infants and children. Report of 12 cases. Clin Pediatr (Phila). 1983;22(6):420-422.

7. Caffey J. On the natural regression of pulmonary cysts during early infancy. Pediatrics. 1953;11(1):48. 